\title{
Specific heat and thermal conductivity in the mixed state of $\mathrm{MgB}_{2}$
}

\author{
L. Tewordt and D. Fay \\ I. Institut für Theoretische Physik, Universität Hamburg, Jungiusstr. 9, 20355 Hamburg, Germany
}

(November 21, 2018)

\begin{abstract}
The specific heat $C$ and the electronic and phononic thermal conductivities $\kappa_{e}$ and $\kappa_{p h}$ are calculated in the mixed state for magnetic fields $H$ near $H_{c 2}$. The effects of supercurrent flow and Andreev scattering of the Abrikosov vortex lattice on the quasiparticles are taken into account. The resulting function $C(H)$ is nearly linear while $\kappa_{e}(H)$ exhibits an upward curvature near $H_{c 2}$. The slopes decrease with impurity scattering which improves the agreement with the data on $\mathrm{MgB}_{2}$. The ratio of phonon relaxation times $\tau_{n} / \tau_{s}=g\left(\omega_{0}, H\right)$ for phonon energy $\omega_{0}$, which is nearly a step function at $\omega_{0}=2 \Delta$ for the BCS state, is smeared out and tends to one for increasing $H$. This leads to a rapid reduction of $\kappa_{p h}(H)$ in $\mathrm{MgB}_{2}$ for relatively small fields due to the rapid suppression of the smaller energy gap.
\end{abstract}

Numerous experiments indicate that the superconducting state of $\mathrm{MgB}_{2}\left(T_{c}=40 \mathrm{~K}\right)$ [1] is a conventional s-wave pairing state mediated by the electron-phonon interaction. Nevertheless, the theoretical explanation of physical quantities is demanding because two different energy gaps are formed where the larger gap $\Delta_{1}$ is associated with the nearly cylindrical $\sigma$-sheets of the Fermi surface and the smaller gap $\Delta_{2}$ with the three-dimensional $\pi$-sheets. Analysis of the temperature dependence of the measured thermal conductivity in the basal plane of $\mathrm{MgB}_{2}$ [2] gives supporting evidence for two different gaps on different Fermi surface sheets. The situation is complicated because the thermal conductivity $\kappa$ is a sum of an electronic part $\kappa_{e}$ and the phononic or lattice conductivity $\kappa_{p h}$. In Ref. [2] $\kappa_{e}$ and $\kappa_{p h}$ were calculated with help of the BCS theory [3] where $\kappa_{e}$ is limited by elastic scattering (scattering by phonons is omitted) and $\kappa_{p h}$ is limited by electron scattering. This theory for $\kappa_{p h}$ is supplemented by adding to the phonon relaxation rate due to scattering by electrons, the relaxation rates due to scattering by point defects [4] and other defects. We remark that the peak in $\kappa_{p h}$ below $T_{c}$ in the cuprate superconductors has been fitted by including scattering by sample boundaries, sheet-like faults, and dislocations [5].

Recently, the ab-plane thermal conductivity of $\mathrm{MgB}_{2}$ has been measured as a function of magnetic field $H$ with orientations both parallel and perpendicular to the c-axis [6]. At low temperatures $\kappa(H)$ drops steeply for increasing $H$ up to a relatively low field which we denote by $H_{c 2}^{(2)}(\sim 1 \mathrm{kOe})$, and then it rises continuously up to $H_{c 2}(\simeq 30$ $\mathrm{kOe}$ for $\mathbf{H} \| \mathbf{c})$. The first drop of $\kappa$ is interpreted as due to the behavior of $\kappa_{p h}(H)$ which is caused by a strong suppression of the smaller gap $\Delta_{2}$ by relatively small fields. This leads to a rapid increase in the number of those quasiparticles that dominate the scattering of phonons [2]. The rapid suppression of the smaller gap can be explained in terms of a two-band model with different energy gaps where the smaller gap is induced by Cooper pair tunneling from the band with the larger gap [7]. The observed increase of $\kappa(H)$ above $H_{c 2}^{(2)}$ is attributed to the increase of $\kappa_{e}(H)$ because $\kappa_{p h}$ has already reached its normal state value [6]. Since this field dependence of $\kappa_{e}(H)$ at low temperatures is qualitatively similar to that of the specific heat coefficient $\gamma(H)$ [8,9], it is concluded that the growth of $\kappa_{e}(H)$ is due to the rapid increase of quasiparticles in the vortex cores associated with the larger energy gap.

In this Letter we present theories for the specific heat and the electronic and phononic thermal conductivities in the vortex state for applied fields near the upper critical field $H_{c 2}$. For simplicity we consider only one isotropic s-wave pairing gap either on a cylindrical Fermi surface with $\mathbf{H} \| \mathbf{c}$, or on a sperical Fermi surface. Our goal is to explain the measured quantities $\gamma(H)$ and $\kappa_{e}(H)$ near $H_{c 2}(\simeq 30 \mathrm{kOe})$, and $\kappa_{p h}(H)$ below the effective upper critical field $H_{c 2}^{(2)}$ $(\sim 1 \mathrm{kOe})$ for the vortex lattice associated with the smaller energy gap. Our theories are based on the normal and anomalous Green's functions $G$ and $F$ obtained from the Gorkov integral equations with kernels given by the product of Abrikosov vortex lattice order parameters $\Delta\left(\mathbf{r}_{1}\right) \Delta^{*}\left(\mathbf{r}_{2}\right)$ and the phase factor due to the magnetic field [10]. These Green's functions depend sensitively on $\sin \theta$ where $\theta$ is the angle between the quasiparticle momentum $\mathbf{p}$ and the magnetic field $\mathbf{H}$. For $\theta \rightarrow 0, G$ and $F$ tend to the Green's functions for a BCS superconductor while, for $\theta \rightarrow \pi / 2$, they take into account the effects of supercurrent flow and Andreev scattering due to the vortex lattice. This theory has been applied previously to calculate $\kappa_{e}(T, H)$ for energy gaps with line nodes like those occuring in the high- $T_{c}$ cuprates and $\mathrm{Sr}_{2} \mathrm{RuO}_{4}$ 11].

A simplified version of the theory in Ref. [10], which has been derived from the Eilenberger equations [12], yields 
the following expression for the spatial average of the density of states:

$$
N(\omega, \theta) / N_{0} \equiv A(\Omega ; \tilde{\Delta}, \theta)=\operatorname{Re}\left[1+\frac{8 \tilde{\Delta}^{2}}{\sin ^{2} \theta}[1+i \sqrt{\pi} z w(z)]\right]^{-1 / 2} .
$$

The quantities appearing in the equation are defined as follows: $z=2[\Omega \tilde{\Delta}+i(\Lambda / v) \Gamma] / \sin \theta$ with $\theta=L(\mathbf{p}, \mathbf{H})$, $\Lambda=(2 e H)^{-1 / 2}, \tilde{\Delta}=\Delta \Lambda / v, \Omega=\omega / \Delta, \tilde{\Delta}^{2}=\left(H_{c 2}-H\right) / 6 \beta_{A} H, \Delta^{2}=\Delta_{0}^{2}(T)\left[1-\left(H / H_{c 2}\right)\right]$, and $w(z)=$ $\exp \left(-z^{2}\right) \operatorname{erfc}(-i z)$. Here $v$ is the Fermi velocity, $\beta_{A}$ the Abrikosov parameter which we take as $1.2, \Delta_{0}(T)$ is the BCS gap, and $\Gamma$ is the normal-state impurity scattering rate. In the Born and unitary scattering limits, $\Gamma$ has to be replaced by $\Gamma A$ or $\Gamma / A$, respectively, where $A(\Omega)$ is calculated self consistently. The specific heat $\mathrm{C}$ is given by $C=N_{0} T \int_{0}^{\infty} d x x^{2} \operatorname{sech}^{2}(x / 2) A(T x / \Delta)$. At low temperatures $(T \ll \Delta), C=\gamma_{s}(H) T$, where the specific heat coefficient $\gamma_{s}(H)$ is proportional to $A(\Omega=0)$. In Fig. 1 we have plotted $A(\Omega=0)$ versus $H / H_{c 2}$ for $\sin \theta=1$ corresponding to a cylindrical Fermi surface and $\mathbf{H} \| \mathbf{c}$, and impurity scattering rates $\delta \equiv \Gamma / \Delta_{0}=0.1,0.2$, and 0.5 . A is seen to be nearly linear near $H_{c 2}$ with a slope at $H_{c 2}$ that decreases for increasing $\delta$. In Fig. 1 we also show our results for the angular average $\bar{A}=\int_{0}^{\pi / 2} d \theta \sin \theta A(\theta)$ which corresponds to a three dimensional Fermi surface. Comparison with the solid curves in Fig. 1 shows that the slopes at $H_{c 2}$ are decreased by the angular average. It should be pointed out that our results are only strictly valid in the vicinity of $H_{c 2}$. However, solution of the Eilenberger equations for a vortex lattice shows that the spatial average of the resulting density of states is well approximated by $A(\Omega)$ for fields down to about $(1 / 2) H_{c 2}$ [13]. The solid curve of $A$ vs $H / H_{c 2}$ for $\delta=0.5$ in Fig. 1 agrees qualitatively with the measured field dependence of the specific heat coefficient $\gamma_{s}(H)$ at low temperatures and fields near $H_{c 2}$ [8.97. In the unitary impurity scattering limit $A$ increases slightly for decreasing field, however at lower fields our theory does not apply anyway.

We turn now to the theory of the electronic thermal conductivity $\kappa_{e}$ in the vortex state near $H_{c 2}$ which has been developed in Ref. [11]. These expressions are easily modified to apply to an isotropic s-wave pairing state and a field along the c-axis. Employing the expression valid at low temperatures we obtain for the ratio $\kappa_{e s} / \kappa_{e n}$ as a function of $H / H_{c 2}$ the plots shown in Fig. 2 for constant $\sin \theta=1$ and impurity scattering rates $\delta=0.1,0.2$, and 0.5. One sees that these plots exhibit upward curvatures with decreasing slopes at $H_{c 2}$ for increasing values of $\delta$. In Fig. 2 we have also plotted the corresponding results for the angular averages over $\theta$ of $\kappa_{e s} / \kappa_{e n}$. Comparison with the measured $\kappa(H)$ near $H_{c 2}$, which is presumably dominated by the field dependence of $\kappa_{e}(H)$ [6], shows that the measured upward curvature towards $H_{c 2}$ is qualitatively best described by the upper solid curve in Fig. 2 for the relatively large impurity scattering rate $\delta=0.5$. It should be noted that the renormalization of the impurity scattering rate in the unitary limit, $\Gamma \rightarrow \Gamma / A$, slightly increases the upward curvature of $\kappa_{e s} / \kappa_{e n}$ in comparison to that obtained without renormalization or in the Born scattering limit. This is in somewhat better agreement with the data. Of course our theory cannot explain the measured field dependence of $\kappa_{e}(H)$ at lower fields which first rises steeply and then saturates until the upward curvature sets in at about $(1 / 2) H_{c 2}$. Our theory also cannot describe the measured anomalous temperature dependence $\kappa_{e} \propto T^{1 / 2}$ for fields below approximately $(1 / 2) H_{c 2}: \kappa_{e n}$ and thus $\kappa_{e s}$ is proportional to $T / \Gamma$.

It was argued in Ref. [6] that the similar field dependencies of the specific heat and electronic thermal conductivity can be explained in terms of the relationship $\kappa_{e}=C_{e} v_{F} \ell / 3$ where $\ell$ is the mean free path. In fact, our theoretical expression for $\kappa_{e}$ has a similar form if one sets $\ell=v_{F} \tau_{e}$ where $\tau_{e}=1 / \operatorname{Im} \xi_{0}$ with $\operatorname{Im} \xi_{0}=\gamma+\gamma_{A}$ [11]. Here $\gamma$ is the impurity scattering rate and $\gamma_{A}$ is the scattering rate due to Andreev scattering of the quasiparticles by the vortices. $\gamma_{A}$ is shown as a function of angle $\theta$ for different values of $\Omega$ and $\tilde{\Delta}$ in Ref. [14]. These plots show that, for extended states $(\Omega \geq 1), \gamma_{A}$ increases from zero as $\theta$ increases from 0 to $\pi / 2$. This means that the Andreev scattering rate has a maximum for quasiparticles moving perpendicular to the vortex axis. For increasing field (decreasing $\tilde{\Delta}$ ), $\gamma_{A}$ decreases which has the effect that the curve for $\kappa_{e s} / \kappa_{e n}$ exhibits an upward curvature in comparison to $C_{e}$ which has a slight downward curvature for increasing field.

Until now there exists only a phenomenological theory for the phonon heat transfer in the mixed state where the phonons are scattered by vortices consisting of normal state cylinders 115]. We develop here the microscopic theory of $\kappa_{p h}$ (limited by electron scattering) in the vortex state in close analogy to the theory for the BCS state [3]. In that case the sum of probabilities for absorption and emission of phonons by quasiparticles yields a relaxtion rate $1 / \tau_{s}$ for a phonon of energy $\omega_{0}$ which is proportional to the integral over quasiparticle energy $E$ of the expression $\left|E E^{\prime} / \varepsilon \varepsilon^{\prime}\right|\left[1-\Delta^{2} / E E^{\prime}\right]\left[f(E / T)-f\left(E^{\prime} / T\right)\right]$. Here $E / \varepsilon=E /\left(E^{2}-\Delta^{2}\right)^{1 / 2}$ is the density of states, $E^{\prime}=E+\omega_{0}$, and $f(E / T)$ is the Fermi function. In the mixed state near $H_{c 2}$, the density of states $E / \varepsilon$ is replaced by $A(\Omega)$ as given in Eq. (11). The coherence term $(\Delta / \varepsilon)\left(\Delta / \varepsilon^{\prime}\right)$, which arises from the matrix elements for quasiparticle scattering, is replaced by $F F^{\dagger}$ where the anomalous Green's function $F$ is given in Ref. [11]. The spectral function of $F$ yields the following analog of $\Delta / \varepsilon$ : 


$$
B(\Omega ; \tilde{\Delta}, \theta)=\operatorname{Re}\left[\frac{-i \sqrt{\pi} 2(\tilde{\Delta} / \sin \theta) w(z)}{\left\{1+\left(8 \tilde{\Delta}^{2} / \sin ^{2} \theta\right)[1+i \sqrt{\pi} z w(z)]\right\}^{1 / 2}}\right] .
$$

The argument $z$ was defined below Eq.(1). The functions $A(\Omega)$ and $B(\Omega)$ are even and odd in $\Omega$. For $\theta \rightarrow 0$ they tend to the BCS functions $\omega /\left(\omega^{2}-\Delta^{2}\right)^{1 / 2}$ and $\Delta /\left(\omega^{2}-\Delta^{2}\right)^{1 / 2}$, respectively. In this way we obtain for the ratio of phonon relaxation times in the normal and superconducting states (denoted by $\mathrm{g}$ in Refs. [2], [3], and [5]):

$$
\begin{aligned}
\tau_{n} / \tau_{s}=g\left(\Omega_{0}\right)= & {\left[1-\exp \left(-\Omega_{0} \Delta / T\right)\right]\left(2 / \Omega_{0}\right) \int_{0}^{\infty} d \Omega f\left[\left(\Omega-\Omega_{0} / 2\right) \Delta / T\right] f\left[-\left(\Omega+\Omega_{0} / 2\right) \Delta / T\right] } \\
& \times\left[A\left(\Omega-\Omega_{0} / 2\right) A\left(\Omega+\Omega_{0} / 2\right)-B\left(\Omega-\Omega_{0} / 2\right) B\left(\Omega+\Omega_{0} / 2\right)\right] .
\end{aligned}
$$

The quantity $\Omega_{0}=\omega_{0} / \Delta$ is the phonon energy $\omega_{0}$ divided by the effective gap $\Delta=\Delta(H)$. For $T \ll \Delta$, the function $f f$ in the integrand of Eq.(3) is approximately one in the range from $\Omega=0$ to $\Omega_{0} / 2$ and zero above $\Omega_{0} / 2$. In Fig. 3 we show some examples of the function $g\left(\Omega_{0}\right)$ for parameter values $\theta=\pi / 2, \delta=0.1$, and $\tilde{\Delta}=0.1,0.2,0.3$, and 0.6 corresponding to $H / H_{c 2}=0.93,0.78,0.61$, and 0.28 . For increasing $\tilde{\Delta}$, or decreasing field, the function $g$ tends to the BCS step function which is zero in the range from $\Omega_{0}=0$ to 2 , and $\pi / 2$ for $\Omega_{0}>2$ (see Fig. 1 of Ref. [5]). The physical meaning of $g$ at low temperatures for the BCS superconductor is that the minimum energy of a phonon for creating a pair of quasiparticles is $\omega_{0}=2 \Delta$. Fig. 3 shows that, for increasing field, the relaxation rate is more and more smeared out and the ratio $\tau_{n} / \tau_{s}$ tends to one. A similar effect occurs for increasing impurity scattering rate $\delta$.

The expression for the phonon thermal conductivity limited by electron scattering and several other scattering processes is given by [5]

$$
\kappa_{p h}=A t^{3} \int_{0}^{\infty} d x \frac{x^{4} \mathrm{e}^{x}}{\left(\mathrm{e}^{x}-1\right)^{2}}\left[1+\alpha t^{4} x^{4}+\beta t^{2} x^{2}+\delta t x+\gamma t x g(x T / \Delta)\right]^{-1} .
$$

Here $x=\omega_{0} / T, t=T / T_{c}$, and the coefficients $A, \alpha, \beta, \delta$, and $\gamma$ refer to scattering by sample boundaries, point defects, sheet-like faults, dislocations, and quasiparticles, respectively. For $t \ll 1$ and $T \ll \Delta$ the argument of $g\left(\Omega_{0}\right)$ in Eq.(1) is approximately equal to zero. Taking the limit $\Omega_{0} \rightarrow 0$ in Eq.(3) and noting that the coherence term B vanishes at $\Omega_{0}=0$, we obtain $g\left(\Omega_{0}=0\right)=\left[A\left(\Omega_{0}=0, \tilde{\Delta}, \theta\right)\right]^{2}$. For constant $\theta=\pi / 2$ this expression yields the field dependence of $\tau_{n} / \tau_{s}$ for the case $\mathbf{H} \| \mathbf{c}$ and a cylindrical Fermi surface. For a three dimensional Fermi surface, which is presumably more appropriate for the quasiparticles associated with the smaller gap in $\mathrm{MgB}_{2}$, we have to multiply $[A(0, \theta)]^{2}$ by $\sin \theta$ and integrate over $\theta$ from 0 to $\pi / 2$. In Fig. 4 we have plotted our results for $g$ at $\theta=\pi / 2$ and for the angular average of $g$ vs $H / H_{c 2}$ for impurity scattering rates $\delta=0.1$ and 0.5 .

The measured $\kappa_{p h}$ at $H=0$ [2] has been analyzed in terms of an expression which is equivalent to our Eq.(传), apart from the term proportional to $\beta$. By inserting two different contributions for the electron scattering term $\gamma$ corresponding to different gaps $\Delta_{1}$ and $\Delta_{2}$, the fitting procedure indicated that the dominant part of the scattering of phonons by electrons is provided by that part of the electronic excitation spectrum experiencing the smaller gap, $\Delta_{2}$. According to the two-band theory of Ref. [7], this smaller gap is suppressed by a relatively small effective upper critical field $H_{c 2}^{(2)}$ which leads to the measured fast drop of $\kappa_{p h}(H)$ as $H$ increases from 0 to $H_{c 2}^{(2)}[6]$. We conclude that the measured field dependence of $\kappa_{p h}(H)$ below $H_{c 2}^{(2)}$ at low temperatures can be explained by inserting in Eq. (4) the values of the various constants obtained for $H=0$ and, for $\tau_{n} / \tau_{s}=g(H)$, the angular average of $g\left(\Omega_{0}=0\right)=\left[A\left(\Omega_{0}=0, \tilde{\Delta}, \theta\right)\right]^{2}$. The behavior of $g(H)$, which determines the form of the fast reduction of $\kappa_{p h}(H)$, depends on the impurity scattering rate $\delta$ (see Fig. 4). We note that the model of phonon scattering by vortices consisting of normal state cores [15] yields a linear dependence of $g$ on $H / H_{c 2}$.

Our results have been derived for an isotropic s-wave gap. Recently an anisotropic s-wave gap $\Delta(\mathbf{p}) / \Delta=(1+$ $\left.a \cos ^{2} \theta\right)^{-1 / 2}$ has been proposed which can explain the anisotropy of $H_{c 2}$ in $\mathrm{MgB}_{2}$ [16]. For this gap with reasonable parameter values of $\mathrm{a}=10$ and 20 we find that the effect of this gap anisotropy on the field dependence of $C$ and $\kappa_{e s} / \kappa_{e n}$ is similar to that of impurity scattering. For instance, the curves of $C$ and $\kappa_{e s} / \kappa_{e n}$ versus $H / H_{c 2}$ for $\delta=0.1$ and $a=20$ appear to yield better fits to the experimental points in Figs. 1 and 2 than the dashed curves for $a=0$ and $\delta=0.5$.

The other important effect on the upward curvature of $\kappa_{e s} / \kappa_{e n}$ arises from the decrease of the Andreev scatering rate $\gamma_{A}$ with increasing field. This decrease is given approximately by the expression $\gamma_{A}=$ $\sqrt{\pi}\left(\tilde{\Delta}^{2} / \sin \theta\right) \exp \left[-(2 \Omega \tilde{\Delta} / \sin \theta)^{2}\right]$ where $\tilde{\Delta}^{2} \sim\left(H_{c 2}-H\right) / H$. It is interesting that the exponent in this expression, in particular the term $(\sin \theta)^{-2}$, is similar to the exponent in the scattering rate $1 / \tau_{v}$ which has been derived 
previously [17]. In that model a quasiparticle is converted into a hole due to Andreev reflection by vortex screening currents. This process corresponds just to the imaginary part of the self energy calculated in Ref. [10].

Our theory is strictly valid only for fields near the upper critical field $H_{c 2}$. However, recent numerical solutions of the quasiclassical Eilenberger equations for the Abrikosov vortex lattice 13 have shown that the analytical expressions for the density of states 10,12 (here the function $A(\Omega)$ ) yield at low energies impressively good results over the whole range of fields down to $H_{c 1}$. These results for low energies are important for the calculation of thermodynamic quantities. We expect that this is also true for the function $B(\Omega)$, the spectral function of the anomalous Green's function.

In conclusion, we have employed microscopic theories for the electronic specific heat $C(H)$ and the thermal conductivity $\kappa_{e}(H)$ and developed a microscopic theory for the phononic thermal conductivity $\kappa_{p h}(H)$ to explain the measured field dependence of these quantities in the mixed state of $\mathrm{MgB}_{2}$. We find that impurity and Andreev scattering, as well as Fermi surface and gap anisotropy, give rise to large effects. For sufficiently large impurity scattering rate, or gap anisotropy, one can approximately fit the data for $C(H)$ and $\kappa_{e}(H)$ for $\mathrm{MgB}_{2}$ over a broad field range below $H_{c 2}$. Our new theory for $\kappa_{p h}(H)$ is capable of describing the observed rapid reduction of the total $\kappa$ of $\mathrm{MgB}_{2}$ for small fields if this is actually caused by suppression of a second smaller energy gap.

We thank T. Dahm for helpful discussions.

[1] J. Nagamatsu, N. Nakagawa, T. Muranaka,Y. Zenitani, and J. Akimitsu, Nature 410, 63 (2001).

[2] A. V. Sologubenko, J. Jun, S. M. Kazakov, J. Karpinski, and H. R. Ott, cond-mat/0111273 (unpublished).

[3] J. Bardeen, G. Rickayzen, and L. Tewordt, Phys. Rev. 113, 982 (1959).

[4] P. G. Klemens and L. Tewordt, Rev. Mod. Phys. 36, No. 1, 118 (1964).

[5] L. Tewordt and Th. Wölkhausen, Solid State Commun. 70, 839 (1989).

[6] A. V. Sologubenko, J. Jun, S. M. Kazakov, J. Karpinski, and H. R. Ott, cond-mat/0201517 (unpublished).

[7] N. Nakai, M. Ichioka, and K. Machida, J. Phys. Soc. Jpn. 71, 23 (2002).

[8] A. Junod, Y. Wang, F. Bouquet, and P. Toulemonde, cond-mat/0106394 (unpublished).

[9] H. D. Yang et al., Phys. Rev. Lett. 87, 167003 (2001).

[10] U. Brandt, W. Pesch, and L. Tewordt, Z. Phys. 201, 209 (1967).

[11] L. Tewordt and D. Fay, Phys. Rev. B 64, 24528 (2001).

[12] W. Pesch, Z. Phys. B 21, 263 (1975).

[13] T. Dahm, S. Graser, C. Iniotakis, and N. Schopohl, cond-mat/0205163 (unpublished).

[14] L. Tewordt and D. Fay, Phys. Rev. B 65, 104510 (2002).

[15] J. B. Sousa, Physica 55, 507 (1971).

[16] A. I. Posazhennikova, T. Dahm, and K. Maki, cond-mat/0204272 v2.

[17] F. Yu, M. B. Salamon, A. J. Leggett, W. C. Lee, and D. M. Ginsberg, Phys. Rev. Lett. 74, 5136 (1995). 
Fig. 1

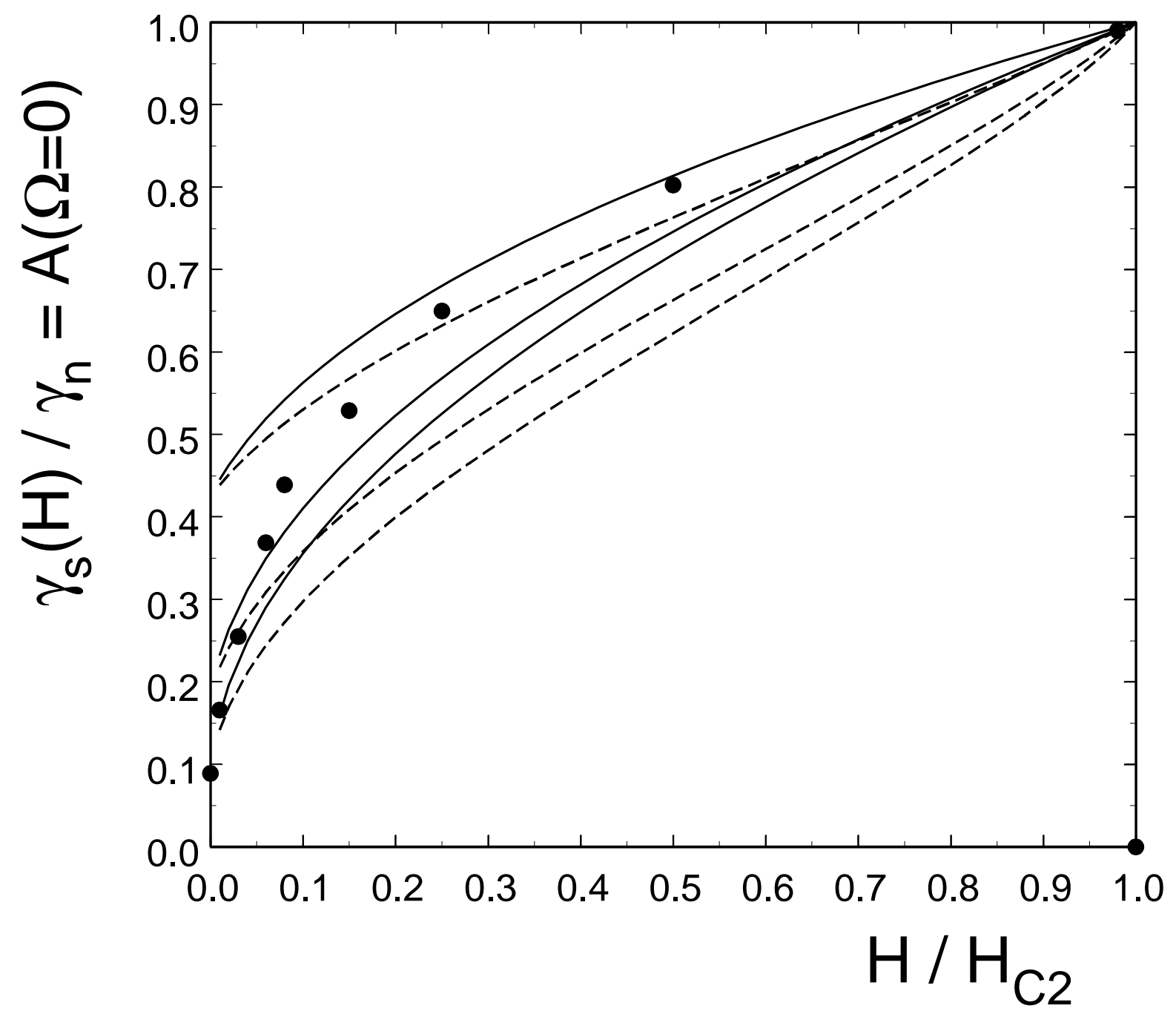

FIG. 1. $A(\Omega=0, H)=\gamma_{s}(H) / \gamma_{n}$ vs $H / H_{c 2}$ for impurity scattering rates $\delta=\Gamma / \Delta_{0}=0.1,0.2$, and 0.5 (from bottom to top). Solid curves for angle $\theta=\pi / 2$ corresponding to $\mathbf{H} \| \mathbf{c}$ for a cylindrical Fermi surface $(\theta$ is the angle between the quasiparticle momentum $\mathbf{p}$ and the field $\mathbf{H}$ ). Dashed curves for the average of $A$ over $\theta$ corresponding to a spherical Fermi surface. The dots are reduced data from Ref. [8] for $\mathrm{MgB}_{2}$ at $T=3 K$. 
Fig. 2

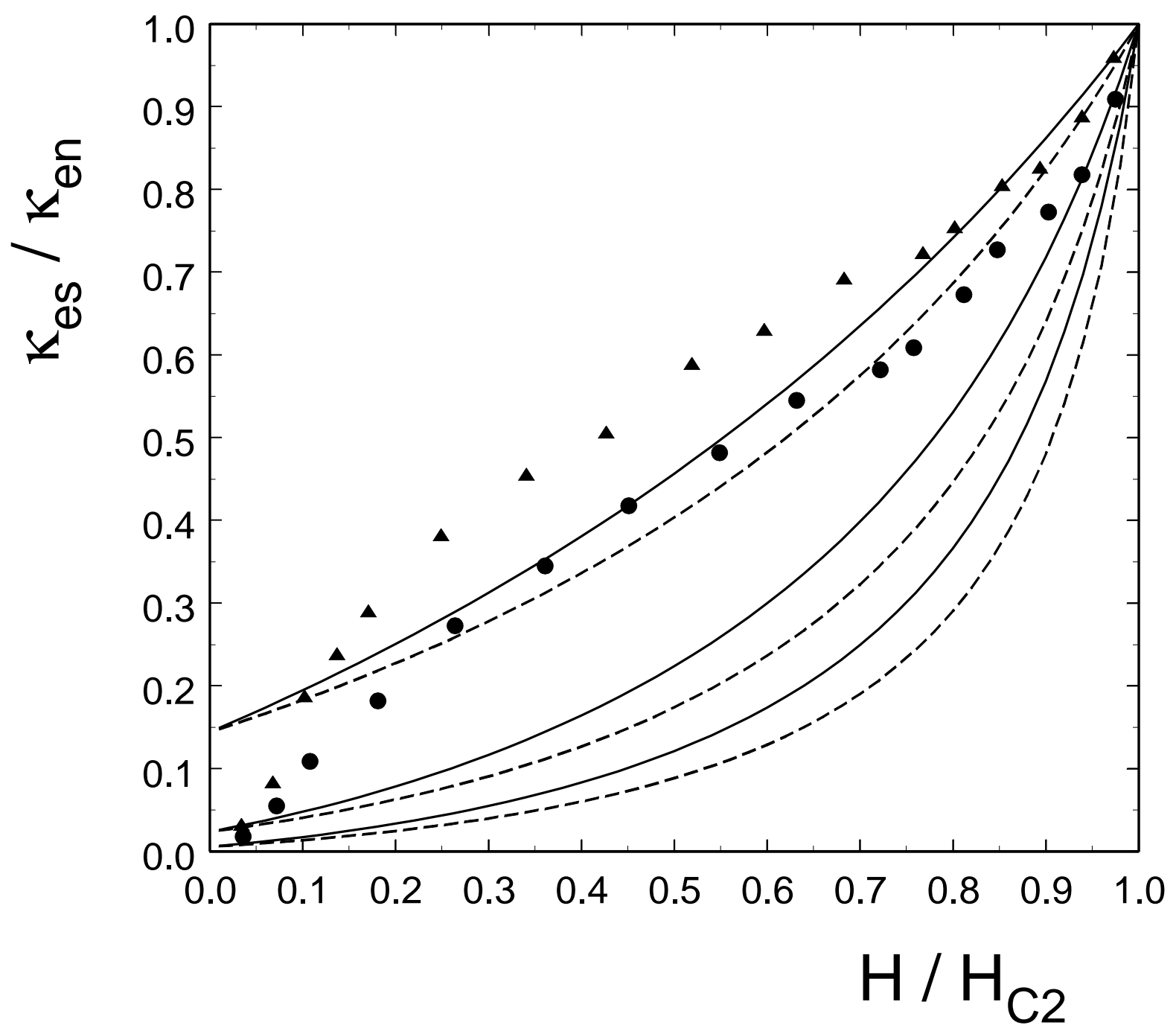

FIG. 2. Electronic thermal conductivity ratio $\kappa_{e s} / \kappa_{e n}$ at low temperatures vs $H / H_{c 2}$ for impurity scattering rates $\delta=0.1$, 0.2 , and 0.5 (from bottom to top). Notation as in Fig.1: Solid curves for angle $\theta=\pi / 2$. Dashed curves for the average over $\theta$. The dots (triangles) are reduced data from Ref. [6] for $\mathrm{MgB}_{2}$ at $T=7.94 K(T=5.13 K)$. 
Fig. 3

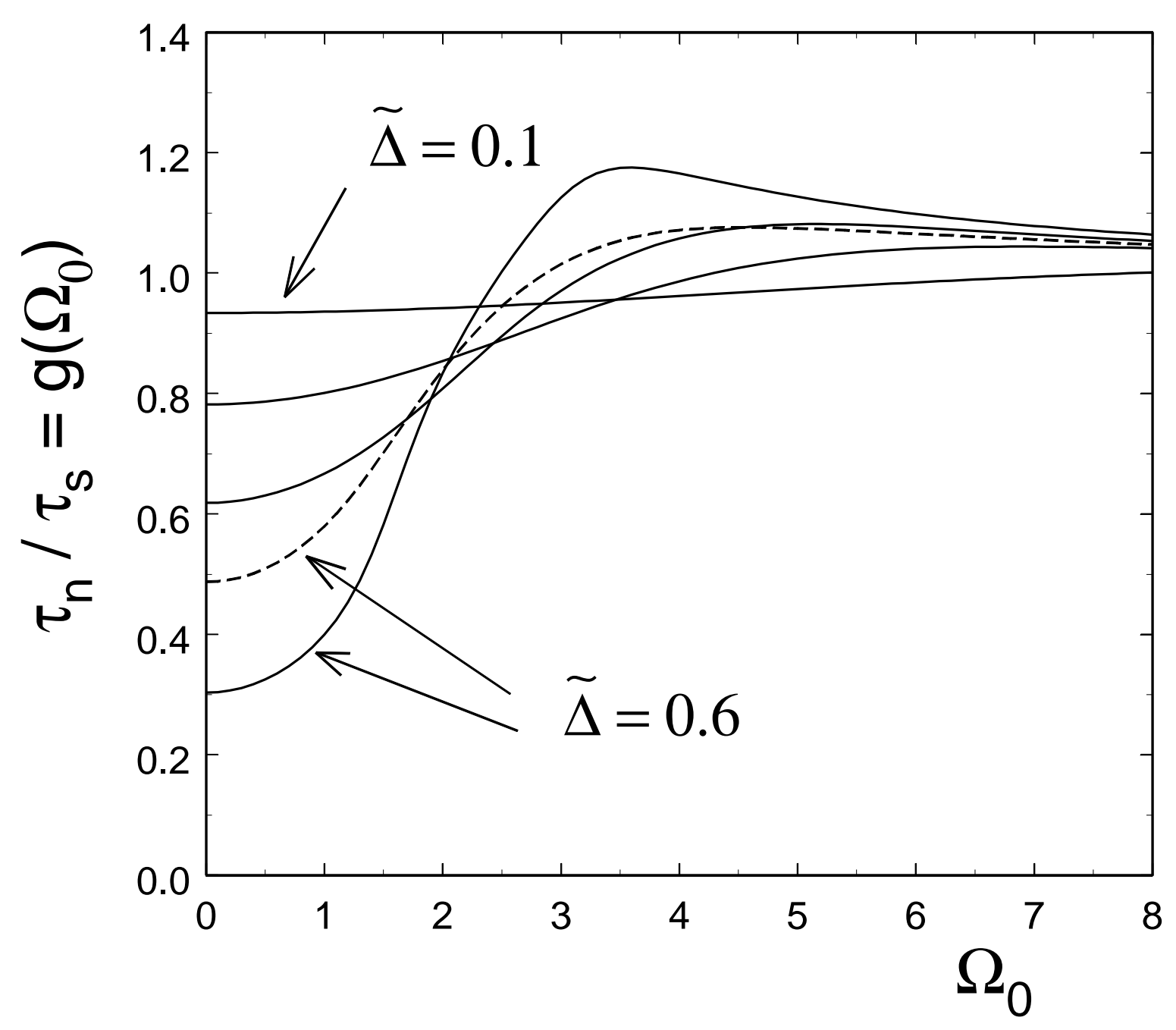

FIG. 3. Ratio of phonon relaxation times $\tau_{n} / \tau_{s}=g\left(\Omega_{0}\right)$ due to electron scattering at low temperatures vs $\Omega_{0}=\omega_{0} / \Delta$ where $\omega_{0}$ is the phonon energy and $\Delta=\Delta(H)$ the effective energy gap. From top to bottom, $\tilde{\Delta}=0.1,0.2,0.3$, and 0.6 , corresponding to $H / H_{c 2}=0.93,0.78,0.61$, and 0.28 . Also, $\delta=0.1$ and $\theta=\pi / 2$ (notation of Fig.1). The dashed curve refers to $\tilde{\Delta}=0.6$ and impurity scattering rate $\delta=0.5$. 
Fig. 4

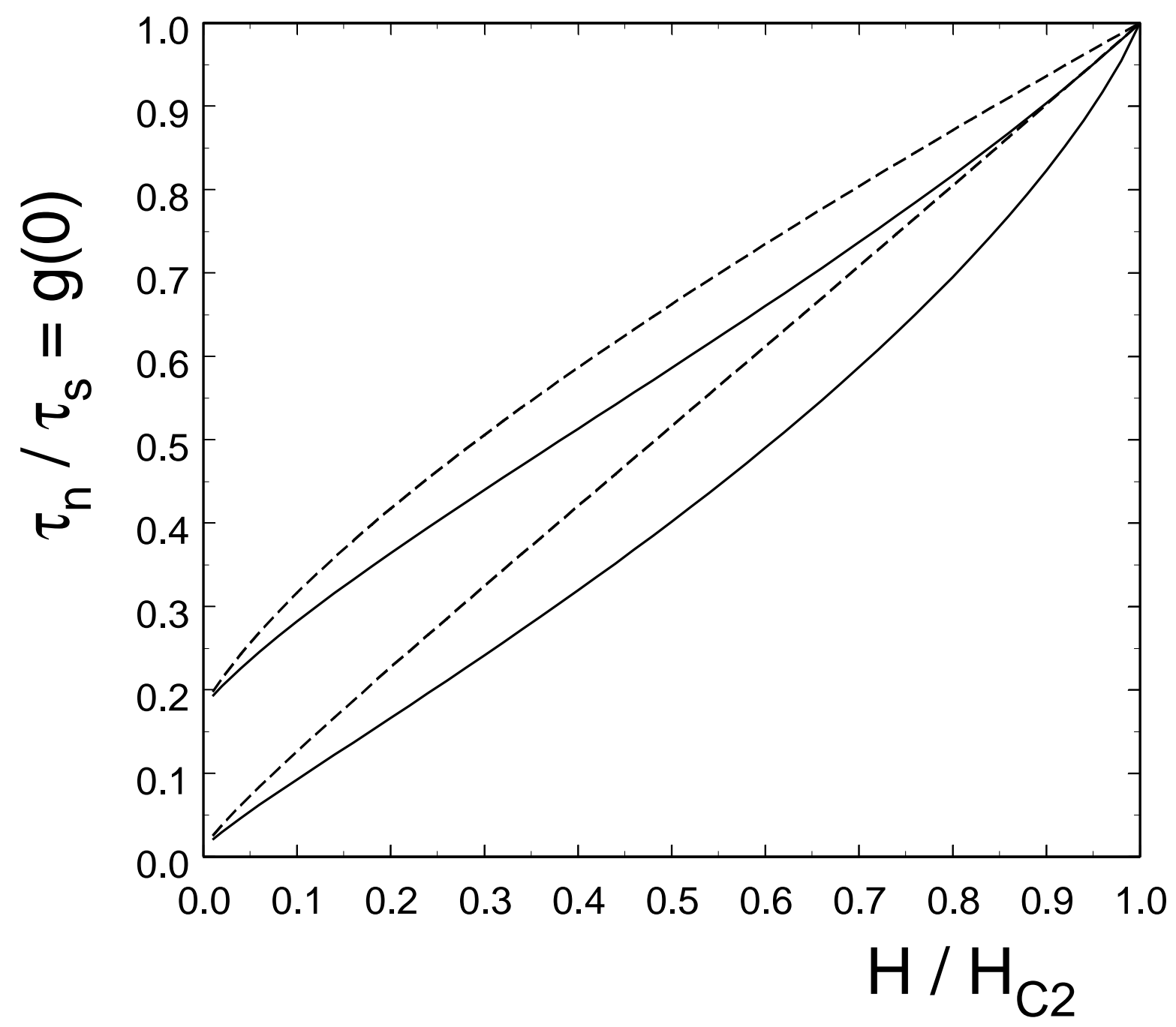

FIG. 4. Ratio $\tau_{n} / \tau_{s}=g\left(\Omega_{0}=0, H\right)$ vs $H / H_{c 2}$ for $\delta=0.1$ (lower curves) and $\delta=0.5$ (upper curves) with the notation of Fig.3. This quantity determines the reduction of the phonon thermal conductivity at low temperatures due to the increase of scattering by quasiparticles. The solid curves are the average of $g$ over $\theta$ corresponding to a spherical Fermi surface and the dashed curves are $g$ for constant $\theta=\pi / 2$. 